\title{
Clinical Outcome of Novel Reconstruction of Double Shouldering Technique after Proximal Gastrectomy
}

\author{
Min Chul Kim, M.S., M.D. ${ }^{1}$, Amir Ben Yehuda, M.D. ${ }^{1}$, Young-Woo Kim, M.D., Ph.D. ${ }^{2,3}$, Hong Man Yoon, M.S., M.D. ${ }^{2}$, \\ Harbi Khalayleh, M.D. ${ }^{2}$, Won Ho Han, M.D. ${ }^{1}$, Hirokazu Noshiro, M.D., Ph.D. ${ }^{4}$ \\ ${ }^{1}$ Department of Surgery, National Cancer Center, ${ }^{2}$ Center for Gastric Cancer, National Cancer Center, ${ }^{3}$ Department of Cancer Control and Population \\ Health, National Cancer Center Graduate School of Cancer Science and Policy, Goyang, Korea, ${ }^{4}$ Department of Surgery, Saga University, Saga, Japan
}

Purpose: Various reconstruction methods have been proposed to reduce reflux after proximal gastrectomy, and we report here a double shouldering technique. The purpose of this study is to compare the novel double shouldering technique with conventional esophagogastrostomy in terms of short term and 3-year clinical outcome.

Methods: A retrospective observational case control study was performed on 63 patients for cT1N0 upper third gastric cancer who underwent proximal gastrectomy from January 2012 to November 2016 at the National Cancer Center, Korea. There were 26 patients with conventional esophagogastrostomy, and 37 patients with novel double shouldering technique. The primary outcome was endoscopic reflux esophagitis findings one and three year after surgery according to Los Angeles classification. Secondary outcomes were short term surgical outcome and reflux symptom.

Results: There was no significant difference in reflux esophagitis on endoscopic findings at 1 and 3 years after surgery between the two group. The double shouldering (DS) technique group showed significantly better postoperative outcomes with bile reflux at one and three years via endoscopic findings versus conventional esophagogastrostomy (CEG). Operative time and hospital stay were significantly shorter in the CEG group than the DS group. There was no significant difference in terms of reflux symptoms and complications.

Conclusion: This novel DS technique is a reconstruction method for use after proximal gastrectomy. It did not show a significant clinical benefit. Development of surgical techniques and further study is needed to identify the optimal reconstruction method after proximal gastrectomy.

Keywords: Gastric cancer, Reconstructive surgical procedures, Gastrectomy

This is an Open Access article distributed under the terms of the Creative Commons Attribution Non-Commercial License (http:/ creativecommons.org/licenses/by-nc/4.0/) which permits unrestricted non-commercial use, distribution, and reproduction in any medium, provided the original work is properly cited.
Received August 31, 2020

Revised 1st October 26, 2020

2nd November 6, 2020

Accepted November 6, 2020

Corresponding author

Young-Woo Kim

Department of Cancer Control and

Population Health, National Cancer

Center Graduate School of Cancer

Science and Policy, 323 Ilsan-ro,

Ilsandong-gu, Goyang 10408, Korea

Tel: $+82-31-920-1635$

Fax: +82-31-920-0696

E-mail: gskim@ncc.re.kr

ORCID:

https://orcid.org/0000-0002-1559-9672

Min Chul Kim and Amir Ben Yehuda contributed equally to this work.

Copyright (C) 2020 The Journal of Minimally Invasive Surgery. All rights reserved.

\section{INTRODUCTION}

For upper third early gastric cancer, total gastrectomy (TG) is still more frequently performed than proximal gastrectomy (PG). As of 2014, only $14.8 \%$ of patients with upper third early gastric cancer underwent proximal gastrectomy in Korea. ${ }^{1}$ Due to the national gastric cancer screening project, the frequency of early gastric cancer is increasing over time. ${ }^{2}$ Early gastric cancer is reported to have a 5 -year survival rate of more than $90 \%$ with curative resection. Therefore, in recent years, the quality of life of patients after surgery has become an important issue, and laparoscopic surgery and function preserving surgery have been widely introduced in this regard. ${ }^{3}$ Total gastrectomy and proximal gastrectomy have not been shown to differ in long-term survival in 
upper third early gastric cancer, and proximal gastrectomy leads to higher absorption of vitamin B12 and other nutrients, reduced anemia, and less weight loss than total gastrectomy. ${ }^{4-6}$

The only reason to prefer total gastrectomy is reflux esophagitis and anastomotic stricture. ${ }^{7}$ Due to these advantages of proximal gastrectomy, there are ongoing attempts to improve the disadvantages of reflux esophagitis through the development of new reconstruction methods including modified esophagogastrostomy after proximal gastrectomy.

The Korean practice guideline for gastric cancer announced in 2019 supports both proximal and total gastrectomy for upper third early gastric cancer in terms of survival, nutrition, and quality of life. ${ }^{8}$ Various reconstruction methods have been introduced after proximal gastrectomy to preserve physiological function with remnant stomach, but there are no general agreements on which method is optimal. ${ }^{9}$

The aim of this study is to compare modified esophagogastrostomy with a double shouldering technique (DS) and conventional esophagogastrostomy (CEG) in terms of postoperative gastroesophageal reflux and safety.

The hypothesis for the primary outcome of this study is that the double shouldering group shows better outcomes versus the conventional esophagogastrostomy group in terms of endoscopic findings based on Los Angeles (LA) classification at one and three years postoperatively.

\section{MATERIALS AND METHODS}

The manuscript is written based on the STROBE guidelines for an accurate and complete report. The indication for proximal gastrectomy was clinical stage T1N0 and size less than $5 \mathrm{~cm}$ in the upper third of the stomach. More than $50 \%$ of the original stomach should remain after surgery.

This study is a retrospective observational case control study. From January 2012 to November 2016, 63 patients underwent proximal gastrectomy at the National Cancer Center, Korea. The patients consisted of 26 patients with conventional esophagogastrostomy, and 37 patients with double shouldering technique. Patients who were converted to total gastrectomy or follow-up loss were excluded. When comparing the postoperative 3-year endoscopic findings, 6 patients ( 2 patients in CEG group, 4 patients in DS group) were excluded because of missing data for the 3-year endoscopic findings.

After proximal gastrectomy, the anastomosis of the esophagus and the residual stomach is stitched. The two sides of the diaphragm and the remnant stomach are then sewn to create two artificial His angles to minimize the reflux symptoms (Fig. 1).

The primary outcome of this study was endoscopic reflux esophagitis findings at one and three years after surgery. The analysis was based on the LA classification results recorded on the endoscopic findings. Secondary outcomes were postoperative complications including mortality and reflux symptoms. When analyzing endoscopic findings, data based on residue, gastritis, bile (RGB) classification was also included, which classified residual food and gastritis into five grades and bile reflux into two grades. Data of reflux symptoms were attained on electronic medical records (EMR) directly or indirectly from the prescription of anti-reflux medication (proton pump inhibitor (PPI), histamine 2 receptor antagonist (H2 blocker), sucralfate, prokinetics) at outpatient clinic. We defined no complaints of reflux symptom as minimal reflux symptom. Mild reflux symptoms refer to occasional symptom occurrence, but self-limiting without hindrance to daily life. The disease was classified as moderate reflux symptom when symptoms resolve with anti-reflux medication and removal from pharmacotherapy is possible. Severe reflux symptoms are defined as a case of ongoing reflux symptoms and relapse of symptoms when discontinuing anti-reflux medication.

The statistical analysis was performed using the SAS software, version 9.4 (SAS institute Inc., Cary, NC, US). A chi-squared test or Fisher's exact test was conducted when comparing the nominal scale. The normality test was performed first in comparison of the two groups with a Kolmogorov Smirnov test and ShapiroWilk test. We accepted the null hypothesis when the $p$ value was above 0.05 assuming normality; the two groups were compared using student t-test. A comparative analysis was conducted with the non-parametric Mann-Whitney test. A Wilcoxon signed rank test was performed to see if there was a significant improvement in endoscopic findings over time. The study was approved by the institutional review board (NCC2015-0225) and followed the principles of Declaration of Helsinki for health research ethics.

\section{RESULTS}

There were no significant differences in sex, age, BMI, size of

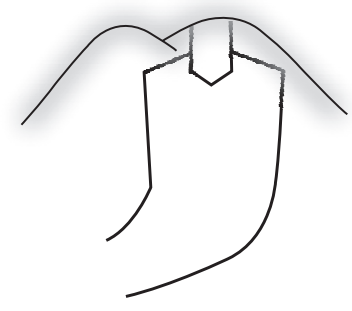

Conventional esophagogastrostomy after proximal gastrectomy

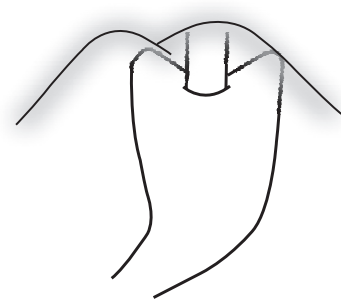

Modified esophagogastrostomy with double shouldering technique
Fig. 1. Schematic of the double shouldering technique: Both sides of proximal part of remnant stomach are anchored to the diaphragm by simple sutures. 
Table 1. Patients demographics \& clinicopathological characteristics

\begin{tabular}{lccc}
\hline & CEG & DS & $p$ value \\
\hline Number of cases & 26 & 37 & \\
Sex & & & 0.723 \\
Male & 20 & 27 & \\
Female & 6 & 10 & \\
Age & $62.5(9.7)$ & $63.6(12.4)$ & 0.562 \\
BMl & $24.0(2.1)$ & $24.1(3.2)$ & 0.986 \\
Size of tumor & $2.5(1.4)$ & $2.9(1.4)$ & 0.244 \\
Location & & & 0.085 \\
High body & 14 & 14 & \\
Cardia & 7 & 20 & \\
Fundus & 5 & 3 & \\
Pathological stage (AJCC 7 th) & & 0.054 \\
la & 23 & 25 & \\
Ib & 2 & 7 & \\
Ila & 1 & 3 & \\
Ilb & 0 & 1 & \\
Illa & 0 & 1 & \\
\hline CEG & & & \\
\hline
\end{tabular}

$\overline{\mathrm{CEG}}=$ conventional esophagogastrostomy group; $\mathrm{DS}=$ double shouldering technique group; $\mathrm{BMl}=$ body mass index.

tumor, location of tumor, and tumor stage between two groups (Table 1). The CEG group showed significantly less operative time and hospital stay than DS group (Table 2). Comparison results for severe complications of Clavien-Dindo classification III and IV showed no significant differences.

When we see a comparison of endoscopic findings at one and three years after surgery, there was no significant difference in LA classification between two groups (Table 3). A significant difference was found in bile reflux at remnant stomach according to RGB classification. The Wilcoxon signed rank test was done to see if there was a significant improvement or aggravation over time, but only residual food was improved in the DS group from postoperative year one to year three. We compared the degree of reflux symptoms based on medical records (Table 4). There was no significant difference between two groups.

\section{DISCUSSION}

We developed a novel reconstruction method after proximal gastrectomy and compared the samples with CEG. In the endoscopic findings, the DS group showed significantly better results than the CEG group only in terms of bile reflux. It did not show better results in surgical outcome and gastroesophageal reflux.

While maintaining the advantages of esophagogastrostomy,
Table 2. Short-term surgical outcomes

\begin{tabular}{lccc}
\hline & CEG & DS & $p$ value \\
\hline Number of cases & 26 & 37 & \\
Operative time (minute) & $196.7 \pm 83.2$ & $230.5 \pm 106.0$ & 0.009 \\
EBL (cc) & $152.5 \pm 507.2$ & $78.1 \pm 175.8$ & 0.537 \\
Hospital stay (day) & $8.5 \pm 13.4$ & $10.1 \pm 13.0$ & 0.005 \\
Postoperative Complications & & & 0.723 \\
(Clavien-Dindo Classification) $^{*}$ & & & \\
I & 0 & 1 & \\
II & 0 & 1 & \\
IIIA & 6 & 10 & \\
IIIB & 0 & 0 & \\
IV & 0 & 0 & \\
Anastomotic complications & & & \\
Anastomotic stricture & $4(15.4 \%)$ & $4(10.8 \%)$ & 0.707 \\
Anastomotic leakage & $1(3.8 \%)$ & $6(16.2 \%)$ & 0.224 \\
\hline
\end{tabular}

$\mathrm{CEG}=$ conventional esophagogastrostomy group; $\mathrm{DS}=$ double shouldering technique group; $\mathrm{EBL}=$ estimated blood loss. ${ }^{*}$ mean $\pm 2 \mathrm{SD}$.

the double shouldering technique is introduced to minimize the disadvantages of reflux esophagitis and gastroesophageal reflux symptoms. The DS technique is composed of the theoretical basis for preventing reflux by securing both ends of the remnant stomach to the diaphragm to form an artificial neo-double His angle ${ }^{10}$ and allowing anastomosis to be placed in the abdominal cavity. ${ }^{11}$ However, the data confirmed that reflux still exists in terms of endoscopic findings and reflux symptoms. Although it was not a statistically significant difference, DS group tended to complain more severe reflux symptom than CEG group. This is most likely due to differences in tumor location, where CEG groups are located more than half in high body and DS groups are often located in cardia. In order to secure proper surgical margin, more esophagus could be resected in the DS group, which may have prevented anastomosis from being located in the abdominal cavity, causing the reflux symptom to be called out due to poor formation of the artificial His angle.

The longer operative time of DS group can be attributed to the addition of the process of fixing the remnant stomach to diaphragm after CEG. The longer hospital stay may be linked to the higher number of postoperative complications. Although there are no statistically significant differences, the greater number of anastomotic leakages in the DS group may have been associated with various early attempts, including the development of new surgical techniques, hand-sewn anastomosis, as well as the introduction of the laparoscopic linear stapler and circular stapler.

To date, the most effective anti-reflux procedure after proximal gastrectomy is double tract reconstruction. Initial studies at 
Table 3. Endoscopic findings on postoperative year one and three

\begin{tabular}{|c|c|c|c|c|c|}
\hline & \multicolumn{2}{|c|}{ CEG } & \multicolumn{2}{|c|}{ DS } & \multirow{2}{*}{$p$ value $^{*}$} \\
\hline & 1 year & 3 year & 1 year & 3 year & \\
\hline Number of cases & 26 & 24 & 37 & 33 & \\
\hline Reflux esophagitis (LA classification) & & & & & 0.348/0.892/0.931/0.288 \\
\hline Normal & $19(73.1 \%)$ & $19(79.2 \%)$ & $31(83.8 \%)$ & $26(78.8 \%)$ & \\
\hline$A$ & $3(11.5 \%)$ & $1(4.2 \%)$ & $2(5.4 \%)$ & $2(6.1 \%)$ & \\
\hline$B$ & $3(11.5 \%)$ & $4(16.7 \%)$ & $2(5.4 \%)$ & $2(6.1 \%)$ & \\
\hline C & $1(3.8 \%)$ & $0(0 \%)$ & $2(5.4 \%)$ & $3(9.1 \%)$ & \\
\hline Bile & & & & & 0.036/0.039/0.317/1.000 \\
\hline 0 & $23(88.5 \%)$ & $21(87.5 \%)$ & $37(100 \%)$ & $33(100 \%)$ & \\
\hline 1 & $3(11.5 \%)$ & $3(12.5 \%)$ & $0(0 \%)$ & $0(0 \%)$ & \\
\hline Food & & & & & 0.697/0.708/0.300/0.015 \\
\hline 0 & $16(61.5 \%)$ & $16(66.7 \%)$ & $20(54.1 \%)$ & $23(69.7 \%)$ & \\
\hline 1 & $2(7.7 \%)$ & $1(4.2 \%)$ & $7(18.9 \%)$ & $3(9.1 \%)$ & \\
\hline 2 & $3(11.5 \%)$ & $1(4.2 \%)$ & $2(5.4 \%)$ & $1(3.0 \%)$ & \\
\hline 3 & $5(19.2 \%)$ & $6(25.0 \%)$ & $8(21.6 \%)$ & $6(18.2 \%)$ & \\
\hline
\end{tabular}

$\mathrm{CEG}=$ conventional esophagogastrostomy group, $\mathrm{DS}=$ double shouldering technique group, LA classification = Los Angeles classification. No data of postoperative 3-year endoscopic result: 2 patients in CEG group and 4 patients in DS group. ${ }^{*} p$ value for comparison of postoperative 1-year result of CEG and DS group/postoperative 3-year result of CEG and DS group/CEG group of 1- and 3-year result/DS group of 1- and 3-year result.

Table 4. Reflux symptoms for postoperative 3 years

\begin{tabular}{lccc}
\hline & CEG & DS & $p$ value \\
\hline Number of cases & 26 & 37 & \\
Reflux Symptom & & & 0.126 \\
$\quad$ Minimal & $11(42.3 \%)$ & $11(29.7 \%)$ & \\
Mild & $3(11.5 \%)$ & $2(5.4 \%)$ & \\
Moderate & $4(15.4 \%)$ & $5(13.5 \%)$ & \\
Severe & $8(30.8 \%)$ & $19(51.4 \%)$ & \\
\hline
\end{tabular}

$\mathrm{CEG}=$ conventional esophagogastrostomy group, $\mathrm{DS}=$ double shouldering technique group. Minimal: no complaints of reflux symptom, Mild: symptom occurs sometimes, but self limited, without hindrance to daily life, Moderate: symptoms resolve with anti-reflux medication, deprescribing of medication is possible, Severe: ongoing reflux symptoms, relapse of symptoms when discontinuing anti-reflux medication.

hospitals that first introduced this procedure reported that the incidence of gastroesophageal reflux symptoms decreased to the level of total gastrectomy. ${ }^{12}$ However, this method is relatively complicated, and the anastomosis occurs three times, and thus the operative time is longer. In addition, there are cases where the endoscope cannot observe the remnant stomach properly after the surgery. During the study period, there were 17 cases of double tract reconstruction after proximal gastrectomy in National
Cancer Center, Korea. Three out of the 17 patients failed their endoscopic observation of the remnant stomach.

After gastrectomy, reflux can be evaluated as symptomatic and endoscopically. Symptoms are subjective and may produce different results depending on how and when the data was collected. The symptoms are also weakly correlated with endoscopic findings and pathologic findings. ${ }^{13}$ Therefore, it is important to confirm the endoscopic findings along with reflux symptoms. Representative methods to objectively evaluate endoscopic findings are the LA classification and residue, gastritis, and bile (RGB) classification. The LA classification was proposed at the World Congress of Gastroenterology in 1994 to classify grades from A to $\mathrm{D}$ according to the extent of esophagitis identified by endoscopy. ${ }^{14}$ The RGB classification was proposed in 2002 by Kubo et al. for an easy and practical classification method that can be used for endoscopic findings after gastrectomy. ${ }^{15}$ They classified the amount of residual food into five grades, the degree and the extent of gastritis into five grades, and bile reflux into two grades. LA classification and RGB classification were used in two of the eight papers analyzed in a review article on the reconstruction method after proximal gastrectomy by Nakamura et al. ${ }^{16-22}$ Only one in eight papers reported results for all three: LA classification, RGB classification, and reflux symptoms. ${ }^{17}$ Cai et al. reviewed the reconstruction method after distal gastrectomy, and LA classification was used in 3 of 8 papers, and 4 of 8 papers 
reported results according to RGB classification. ${ }^{23-28}$ Again, only one in eight papers reported results for all three: LA classification, RGB classification, and reflux symptom. ${ }^{26}$

The limitations of this study are that it is a retrospective observational study, that only three surgeons in a single center participated, and there was a small sample size as an observational study. Additionally, we did not find strong evidence to prove safety and effectiveness of the new surgical technique. We have experienced laparoscopy assisted total gastrectomy. ${ }^{29}$ Though clinically acceptable and better quality of life outcome than conventional open total gastrectomy in our assessment, partial gastrectomy preserving healthy part of distal stomach must be beneficial and preferred from most of patients. ${ }^{20}$ Reflux is one of drawback of the proximal gastrectomy but it should not be a rationale for preferring total gastrectomy. Recently developed SPADE operation, devised version of double shouldering technique, showed potential to decrease gastroesophageal reflux after proximal gastrectomy with fair short-term operative outcome. ${ }^{30}$ A prospective clinical study is on planning for this novel procedure. Since proximal gastrectomy is a treatment for gastric cancer, it is necessary to confirm the data through long-term studies in terms of survival and oncologic safety. In addition, long-term studies that compare the nutritional aspects with total gastrectomy are needed. Future studies should compare modified esophagogastrostomy (EG) to total gastrectomy (TG) or double tract (DT) reconstruction method. Once the safety and feasibility of the new modified EG is secured, it is necessary to obtain a higher level of evidence through multicenter prospective controlled studies.

In conclusion, a novel double shouldering technique did not show better clinical outcomes versus conventional simple esophagogastrostomy. Development of this surgical technique and further study is needed to find the optimal reconstruction method after proximal gastrectomy.

\section{ORCID}

Min Chul Kim, https://orcid.org/0000-0002-6596-3161

Amir Ben Yehuda, https://orcid.org/0000-0001-9745-4735

Young-Woo Kim, https://orcid.org/0000-0002-1559-9672

Hong Man Yoon, https://orcid.org/0000-0002-6218-7080

Harbi Khalayleh, https://orcid.org/0000-0002-6706-3837

Won Ho Han, https://orcid.org/0000-0002-7835-7603

Hirokazu Noshiro, https://orcid.org/0000-0003-3227-7816

\section{AUTHORS' CONTRIBUTIONS}

Study concept and design: YK, MK, AY

Acquisition of data, statistical analysis, preparation of the manuscript, and typing: MK, YK
Interpretation of data and critical revision: $\mathrm{HY}, \mathrm{HK}, \mathrm{WH}, \mathrm{HN}$ Final approval: all authors.

\section{CONFLICT OF INTEREST}

None.

\section{FUNDING}

This work was supported by grant NCC- NCC-1710120, 20100901 from the National Cancer Center, Korea.

\section{ACKNOWLEDGMENTS}

None.

\section{REFERENCES}

1) Park DJ, Lee $Y$, Ahn S-H, Kim H-H. Laparoscopic proximal gastrectomy for upper third early gastric cancer. Ann Laparosc Endosc Surg 2017;2:80.

2) Information Committee of Korean Gastric Cancer Association. Korean Gastric Cancer Association Nationwide Survey on Gastric Cancer in 2014. J Gastric Cancer 2016;16:131-140.

3) Kim YW, Baik YH, Yun YH, et al. Improved quality of life outcomes after laparoscopy-assisted distal gastrectomy for early gastric cancer: results of a prospective randomized clinical trial. Ann Surg 2008;248: 721-727.

4) Yoo $\mathrm{CH}$, Sohn $\mathrm{BH}$, Han WK, Pae WK. Long-term results of proximal and total gastrectomy for adenocarcinoma of the upper third of the stomach. Cancer Res Treat 2004;36:50-55.

5) Tanioka T, Waratchanont R, Fukuyo R, et al. Surgical and nutritional outcomes of laparoscopic proximal gastrectomy versus total gastrectomy: a meta-analysis. Surg Endosc 2020;34:1061-1069.

6) Ko HJ, Kim KH, Lee SH, et al. Can Proximal Gastrectomy with Double-Tract Reconstruction Replace Total Gastrectomy? A Propensity Score Matching Analysis. J Gastrointest Surg 2020;24:516-524.

7) Ahn SH, Lee JH, Park DJ, Kim HH. Comparative study of clinical outcomes between laparoscopy-assisted proximal gastrectomy (LAPG) and laparoscopy-assisted total gastrectomy (LATG) for proximal gastric cancer. Gastric Cancer 2013;16:282-289.

8) Guideline Committee of the Korean Gastric Cancer Association (KGCA), Development Working Group \& Review Panel. Korean Practice Guideline for Gastric Cancer 2018: an Evidence-based, Multi-disciplinary Approach. J Gastric Cancer 2019;19:1-48.

9) Shaibu Z, Chen Z, Mzee SAS, Theophilus A, Danbala IA. Effects of reconstruction techniques after proximal gastrectomy: a systematic review and meta-analysis. World J Surg Oncol 2020;18:171.

10) Fujiwara $Y$, Nakagawa K, Kusunoki M, Tanaka T, Yamamura T, Utsunomiya J. Gastroesophageal reflux after distal gastrectomy: possible 
significance of the angle of His. Am J Gastroenterol 1998;93:11-15.

11) Ayazi S, DeMeester SR, Hsieh CC, et al. Thoraco-abdominal pressure gradients during the phases of respiration contribute to gastroesophageal reflux disease. Dig Dis Sci 2011;56:1718-1722.

12) Ahn SH, Jung DH, Son SY, Lee CM, Park DJ, Kim HH. Laparoscopic double-tract proximal gastrectomy for proximal early gastric cancer. Gastric Cancer 2014;17:562-570.

13) Johnsson F, Joelsson B, Gudmundsson K, Greiff L. Symptoms and endoscopic findings in the diagnosis of gastroesophageal reflux disease. Scand J Gastroenterol 1987;22:714-718.

14) Lundell LR, Dent J, Bennett JR, et al. Endoscopic assessment of oesophagitis: clinical and functional correlates and further validation of the Los Angeles classification. Gut 1999;45:172-180.

15) Kubo M, Sasako M, Gotoda T, et al. Endoscopic evaluation of the remnant stomach after gastrectomy: proposal for a new classification. Gastric Cancer 2002;5:83-89.

16) Nakamura $M$, Yamaue $H$. Reconstruction after proximal gastrectomy for gastric cancer in the upper third of the stomach: a review of the literature published from 2000 to 2014. Surg Today 2016;46:517527.

17) Yasuda A, Yasuda T, Imamoto H, et al. A newly modified esophagogastrostomy with a reliable angle of His by placing a gastric tube in the lower mediastinum in laparoscopy-assisted proximal gastrectomy. Gastric Cancer 2015;18:850-858.

18) Nakamura M, Nakamori $M$, Ojima $T$, et al. Reconstruction after proximal gastrectomy for early gastric cancer in the upper third of the stomach: an analysis of our 13-year experience. Surgery 2014;156: 57-63.

19) Nomura E, Lee SW, Kawai M, et al. Functional outcomes by reconstruction technique following laparoscopic proximal gastrectomy for gastric cancer: double tract versus jejunal interposition. World J Surg Oncol 2014;12:20.

20) Masuzawa T, Takiguchi S, Hirao M, et al. Comparison of perioperative and long-term outcomes of total and proximal gastrectomy for early gastric cancer: a multi-institutional retrospective study. World J Surg 2014;38:1100-1106.

21) Takagawa R, Kunisaki C, Kimura J, et al. A pilot study comparing jejunal pouch and jejunal interposition reconstruction after proximal gastrectomy. Dig Surg 2010;27:502-508.

22) Sakuramoto S, Yamashita K, Kikuchi S, et al. Clinical experience of laparoscopy-assisted proximal gastrectomy with Toupet-like partial fundoplication in early gastric cancer for preventing reflux esophagitis. J Am Coll Surg 2009;209:344-351.

23) Cai Z, Zhou Y, Wang C, et al. Optimal reconstruction methods after distal gastrectomy for gastric cancer: A systematic review and network meta-analysis. Medicine (Baltimore) 2018;97:e10823.

24) So JB, Rao J, Wong AS, et al. Roux-en-Y or Billroth II Reconstruction After Radical Distal Gastrectomy for Gastric Cancer: A Multicenter Randomized Controlled Trial. Ann Surg 2018;267:236-242.

25) Hirao M, Takiguchi S, Imamura $\mathrm{H}$, et al. Comparison of Billroth I and Roux-en-Y reconstruction after distal gastrectomy for gastric cancer: one-year postoperative effects assessed by a multi-institutional RCT. Ann Surg Oncol 2013;20:1591-1597.

26) Nakamura M, Nakamori M, Ojima T, et al. Randomized clinical trial comparing long-term quality of life for Billroth I versus Roux-en-Y reconstruction after distal gastrectomy for gastric cancer. Br J Surg 2016;103:337-347.

27) Yang K, Zhang WH, Liu K, Chen XZ, Zhou ZG, Hu JK. Comparison of quality of life between Billroth-I and Roux-en-Y anastomosis after distal gastrectomy for gastric cancer: A randomized controlled trial. Sci Rep 2017;7:11245.

28) Yang D, He L, Tong WH, Jia ZF, Su TR, Wang Q. Randomized controlled trial of uncut Roux-en-Y vs Billroth II reconstruction after distal gastrectomy for gastric cancer: Which technique is better for avoiding biliary reflux and gastritis? World J Gastroenterol 2017;23: 6350-6356.

29) Han WH, Oh YJ, Eom BW, Yoon HM, Kim YW, Ryu KW. A comparative study of the short-term operative outcome between intracorporeal and extracorporeal anastomoses during laparoscopic total gastrectomy. Surg Endosc 2020 Apr 8 [Epub]. https://doi.org/10.1007/ s00464-020-07539-y.

30) Han WH, Eom BW, Yoon HM, Ryu J, Kim YW. Spade-Shaped Anastomosis Following a Proximal Gastrectomy Using a Double Suture to Fix the Posterior Esophageal Wall to the Anterior Gastric Wall (SPADE Operation): Case-Control Study of Early Outcomes. J Gastric Cancer 2020;20:72-80. 